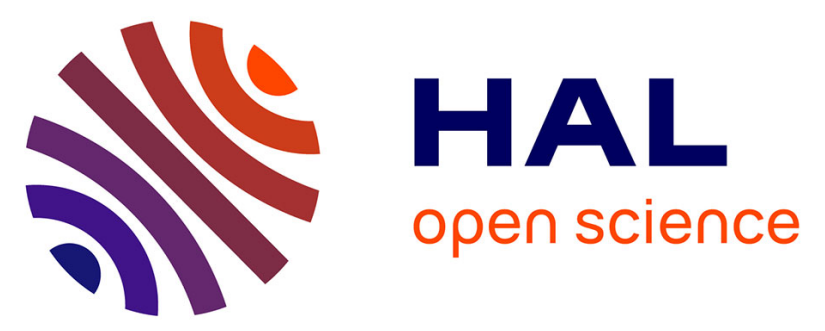

\title{
Longitudinal and transverse hyperpolarizabilities of carbon nanotubes: a computational investigation through the coupled-perturbed Hartree-Fock/Kohn-Sham scheme
}

Valentina Lacivita, Michel Rérat, Roberto Orlando, Roberto Dovesi, Philippe

D'arco

\section{To cite this version:}

Valentina Lacivita, Michel Rérat, Roberto Orlando, Roberto Dovesi, Philippe D'arco. Longitudinal and transverse hyperpolarizabilities of carbon nanotubes: a computational investigation through the coupled-perturbed Hartree-Fock/Kohn-Sham scheme. Theoretical Chemistry Accounts: Theory, Computation, and Modeling, 2016, 135 (3), pp.81. 10.1007/s00214-016-1835-7 . hal-01292442

\section{HAL Id: hal-01292442 https://hal.sorbonne-universite.fr/hal-01292442}

Submitted on 23 Mar 2016

HAL is a multi-disciplinary open access archive for the deposit and dissemination of scientific research documents, whether they are published or not. The documents may come from teaching and research institutions in France or abroad, or from public or private research centers.
L'archive ouverte pluridisciplinaire HAL, est destinée au dépôt et à la diffusion de documents scientifiques de niveau recherche, publiés ou non, émanant des établissements d'enseignement et de recherche français ou étrangers, des laboratoires publics ou privés. 


\title{
Longitudinal and transverse hyperpolarizabilities of Carbon nanotubes. A computational investigation through the Coupled Perturbed Hartree-Fock/Kohn-Sham scheme.
}

\author{
Valentina Lacivita - Michel Rérat - Roberto Orlando • Roberto Dovesi • \\ Philippe D'Arco
}

Received: date / Accepted: date

\begin{abstract}
Static electronic polarizability $\alpha$ and second hyperpolarizability $\gamma$ of semiconduting and conducting carbon nanotubes with radius up to $7.5 \AA$ are evaluated using the coupled-perturbed Hartree-Fock/KohnSham scheme, as implemented in the periodic CRYSTAL14 code, and a split-valence basis set. Two density functionals, namely LDA (pure local) and B3LYP (hybrid), and the Hartree-Fock hamiltonian are compared. A few PBE (gradient corrected) density functional data are also produced for comparison with previous calculations. Convergence of both longitudinal $(L)$ and transverse $(T)$ components is documented. It is shown how the second hyperpolarizability depends critically on the computational conditions, the more so the larger the radius of the nanotube (and thus the smaller the energy gap). The longitudinal component is sensibly affected by the truncation of the exact exchange series (HF and
\end{abstract}

\section{Lacivita}

Institut Calcul et Simulation and Institut des Sciences de la Terre Paris, UPMC, Sorbonne Universités, F-75005 Paris (France)

E-mail: valentina.lacivita@upmc.fr

M. Rérat

Equipe de Chimie Physique, IPREM UMR5254, Université de Pau, 64000 Pau, France

R. Orlando

Dipartimento di Chimica IFM, Università di Torino and NIS - Nanostructured Interfaces and Surfaces - Centre of Excellence, Via P. Giuria 7, 10125 Torino, Italy

\section{R. Dovesi}

Dipartimento di Chimica IFM, Università di Torino and NIS - Nanostructured Interfaces and Surfaces - Centre of Excellence, Via P. Giuria 7, 10125 Torino, Italy

Ph. D'Arco

Institut des Sciences de la Terre Paris (UMR 7193 UPMC-CNRS), UPMC, Sorbonne Universités, F-75005 Paris (France)
B3LYP), which must include electron-electron interactions at a distance up to $100 \AA$ in order to have $\gamma_{L}$ converged to better than $1 \%$. The transverse $\gamma_{T}$ component of conducting tubes critically depends on the number of $\mathbf{k}$ points in reciprocal space: at least $900 \mathbf{k}$ points are required to converge better than $1 \%$ at the LDA level. Coupled-perturbed results are compared to uncoupled values obtained from a sum-over-states (SOS) approach. The difference between the two is particularly important along the transverse direction and when pure DFT functionals are used: the coupled-perturbed correction can shrink the SOS value by several hundreds times. The ratio LDA/HF is roughly constant around 2 for $\alpha_{L}$; it ranges between 25 and 60 for $\gamma_{L}$. As regards the convergence with the nanotube radius, the $R^{2}$ law is confirmed for $\alpha_{L}$ and $\alpha_{T}$ (normalized for the cell parameter) at all levels of theory. For the second hyperpolarizabilities $\gamma_{L}$ and $\gamma_{T}$, a clear $R^{5}$ dependence is observed.

Keywords Carbon nanotubes, polarizability, hyperpolarizability, CPHF, CPKS, Hartree-Fock, density functional, LDA, GGA, hybrid, DFT overshoot, Gaussian basis set, CRYSTAL code

\section{Introduction}

All carbon nanotubes (CNTs) are electrically conductive. However, their electronic structure varies depending on the chiral vector $(m, n)(m, n \in \mathbb{N})$ : as regards the two important families of armchair $(m, m)$ and zigzag $(m, 0)$ CNTs, the former are $1 D$ conductors; the latter are semiconductors with almost vanishing band gap for $m=3 l(l \in \mathbb{N})$. These features, combined with quantum confinement at the nanoscale, originate outstanding optical properties. 
Over the past two decades, several theoretical investigations were carried out concerning the linear dielectric response of CNTs [1-6], including a previous work by some of the present authors [7], based on stateof-the-art coupled-perturbed (CP) Hartree-Fock (HF) and Kohn-Sham (KS) ab initio calculations with large Gaussian basis sets. Results obtained on a number of zig-zag and armchair single-wall CNTs with radius $R$ up to $16.3 \AA$, showed the transverse polarizability $\alpha_{T}^{a}$ (normalized for the cell parameter $a$ ) to scale linearly with the square of the tube radius $R$, while the longitudinal polarizability $\alpha_{L}^{a}$ scales linearly with $R / E_{g}$, where $E_{g}$ is the energy gap.

In recent years, investigations have been extended also to the optical nonlinearity of CNTs [8]. The literature on this topic is abundant on both the experimental and theoretical sides [9-13], but still remains incomplete on many aspects. The properties of interest are the first and second hyperpolarizabilities, $\beta$ and $\gamma$. These are microscopic tensor properties related to the macroscopic dielectric susceptibilities of second $\left(\chi^{(2)}\right)$ and third order $\left(\chi^{(3)}\right)$, respectively, through the cell volume $V$ (for a $3 \mathrm{D}$ system): $\chi^{(2)}=\frac{2 \pi}{V} \beta$ and $\chi^{(3)}=\frac{2 \pi}{3 V} \gamma$.

The inversion center annihilates the first hyperpolarizability $\beta$ of armchair and zig-zag nanotubes, not that of general $(m, n)$ tubes. The space symmetry, given by the product of the $D_{n}$ point symmetry and the axial group of monodimensional translations [14], produces two nonzero $\beta$ components, namely $\beta_{x y z}=-\beta_{y z x}$. At the static limit, however, the additional tensor permutation symmetry [15], implies $\beta_{x y z}(0 ; 0,0)$ to be null. Guo et al. [3] calculated "unscreened" (i.e., sum-overstates, SOS) static and dynamic electronic contributions for small chiral nanotubes $(R<6 \AA)$, using the local-density approximation (LDA) [16] to the density functional theory (DFT). They found the static value of $\chi_{x y z}^{(2)}(0 ; 0,0)$ to be zero indeed, and an absolute value of the second harmonic generation (SHG) coefficient $\chi_{x y z}^{(2)}(-2 \omega ; \omega, \omega)$ as large as $15 \times 10^{-6}$ esu in the photon energy range $0.1-4.0 \mathrm{eV}$. They also noted that the SHG coefficient $\chi_{x y z}^{(2)}(-2 \omega ; \omega, \omega)$ becomes smaller with increasing $R$ as a consequence of the convergence to graphene, that is center-symmetric.

Second-order optical nonlinearity effects were evaluated by Margulis et al. [17] for semiconductor singlewall CNTs. By using a simple two-band approximation, the authors obtained an expression for the static electronic contribution to $\chi^{(3)}$ along the tube axis, that is proportional to the fourth power of the radius $R$, i.e., $\chi_{L}^{(3)}(0 ; 0,0,0) \propto R^{4}$. For a single-wall CNT with radius around $5.5 \AA$, they estimated $\chi_{L}^{(3)}(0 ; 0,0,0)$ to be about $3 \times 10^{-9} \mathrm{esu}$, which is comparable with the largest thirdorder dielectric susceptibility $\chi^{(3)}$ observed for III-V semiconductor compounds. An independent estimate of the static second hyperpolarizability $\gamma_{L}$ of semiconductor CNTs was later provided by Kozinsky and Marzari [5], by applying the numerical finite-field method at the PBE [18] DFT level. For a zig-zag nanotube $(8,0)$ of $3.15 \AA$ radius, they obtained $\gamma_{L}=3.1 \times 10^{7}$ a.u. That is, $\chi_{L}^{(3)} \approx 1 \times 10^{-9}$ esu, if we define an approximate volume $V=\pi R^{2} a$, with $a \approx 4 \AA$ the cell parameter. Although this result is not directly comparable with that obtained by Margulis et al., based on the proposed $R^{4}$ law, the ratio between the given radii, i.e., $5.5 \AA[17]$ and $3.15 \AA[5]$, would lead to guess a difference of about one order of magnitude between the corresponding values of $\chi_{L}^{(3)}$, which is not the case for the data provided by the authors. Further analysis is required in order broaden the spectrum of available absolute data and thus establish reliable trends.

In this paper, we aim to complement and improve upon the above mentioned theoretical results. As a development of our previous study [7], we report about $a b$ initio static electronic hyperpolarizability calculations on CNTs of increasing radius, performed using the coupled-perturbed Hartree-Fock/Kohn-Sham (CPHF / $\mathrm{KS}$ ) computational scheme, as implemented in the quantum chemistry software package for periodic systems CRYSTAL14 [19-24]. A development version of this code is now under test, which also implements the CP calculation of dynamic SHG coefficients. These features will be investigated in a forthcoming paper.

Unlike SOS perturbative methods, as those applied in Refs. [3] and [17], the coupled-perturbed approach fulfills a self-consistent computational procedure to account for orbital relaxation effects induced by the electric field, thus providing far more accurate optical properties. Furthermore, the analytical character of the CP equations largely overcomes, for both accuracy and efficiency, the variational finite-field approach used in Ref. [5]. We will compare $\mathrm{HF}$ and various density functionals, namely conventional local density (LDA) and generalized gradient (PBE) approximations, and the hybrid B3LYP functional. Periodic CPKS calculations represent a severe test of the methodology. It is known that DFT methods may significantly overshoot the electronic (hyper)polarizabilities of extended $\pi$-conjugated systems. An example is that of polyenes [25,26], for which only slight (or even no) convergence towards the periodic limit is found [27]. However, we will show that there is no such overshoot in the case of CNTs, as indicated by a fairly good agreement with the experimental data.

We describe here a robust procedure for the evaluation of the optical properties of CNTs, even in cases where the calculated values blow-up as $E_{g} \rightarrow 0$. In do- 
ing so, we will show that it is possible to draw regular trends with increasing $R$ at every level of theory, regardless of the convergence rates and of the magnitude of the numbers to be managed.

The paper is organized as follows. Computational details are illustrated in Section 2, with particular focus on the most critical parameters, i.e., the number of $\mathbf{k}$ points at which the SCF and CP equations are solved and the thresholds controlling the truncation of the exact exchange series. Results are presented in Section 3, dealing with a) the trends of $\alpha$ and $\gamma$ with increasing radius; b) the comparison between the various levels of theory. Finally, in the last section, a few conclusions are drawn.

\section{Computational details}

Calculations were performed with the periodic $a b$ initio CRYSTAL14 code [19] by using an all-electron splitvalence $6-31 \mathrm{G}(\mathrm{d})$ basis set. Two density functionals, LDA [28], and B3LYP [29] (containing 20\% of exact exchange) are compared with Hartree-Fock (HF). Some calculations have been performed also with the PBE functional, which was previously shown to behave similarly to LDA for the kind of properties investigated here [27].

The geometry was optimized in all cases. The threshold for the convergence of the self-consistent-field (SCF) energy was set to $10^{-10} \mathrm{Ha}$. The density functional exchange-correlation contribution to the total energy was computed using a pruned grid (keyword XLGRID [19]) for numerical integration. Its accuracy can be estimated from the error in the electronic charge per unit cell: 4 $\times 10^{-4}|e|$ for a total number of $240|e|$ for the $(10,0)$ nanotube. To exit the self-consistent $\mathrm{CPHF} / \mathrm{KS}$ process, the variation of $\alpha(\gamma)$ calculated at two subsequent cycles must be smaller than $10^{-2}$ a.u. (1 a.u.).

$\mathrm{CPHF} / \mathrm{KS}$ results for small (null) band gap systems are strongly affected by the shrinking factor $S$ used for sampling the reciprocal space, and by the truncation of the infinite exact exchange series ( $\mathrm{HF}$ and B3LYP cases), as previously documented for polyacetylene [27]. Convergence becomes more critical for large $R$ tubes, because the energy gap narrows $\left(E_{g} \propto 1 / R\right)$ and polarization effects increase. To describe the effect of the aforementioned computational parameters on the calculated optical properties, we will take as references the $(17,0)$ and $(11,11)$ tubes. These are representatives of semiconducting and conducting CNTs, respectively, and have the largest radii here considered. For the $(11,11)$ CNT we will investigate the convergence of $\alpha_{T}$ and $\gamma_{T}$, the longitudinal components being infinite as the solution is metallic. For the $(17,0) \mathrm{CNT}$, instead, we will focus on $\alpha_{L}$ and $\gamma_{L}$ which are finite although very large, whereas the transverse components converge much faster.

\subsection{Shrinking factor}

A dense k-point sampling of the Brillouin zone (BZ) is crucial for the accurate description of the electronic structure around the region where the gap is small. This justifies the need for using a large shrinking factor $S$ to achieve convergence on the calculated optical properties. Pure density functionals (e.g., LDA and GGA) are particularly concerned since they are known to systematically underestimate the band gap. At the other extreme HF overestimates $E_{g}$. Table 1 shows the dependence on $S$ of the (hyper)polarizabilities $\alpha^{C}$ and $\gamma^{C}$ (normalized to one $\mathrm{C}$ atom), calculated at both the LDA and HF levels of theory. For the $(17,0)$ tube, HF provides an energy gap about four times larger than LDA, producing $\alpha_{L}^{C}$ and $\gamma_{L}^{C}$ values that are about 2 and 60 times smaller. All HF and LDA properties are converged to better than $1 \%$ at $S=80$ (41 k points). A much slower convergence with $S$ is found for the transverse properties of the $(11,11)$ tube, that is metallic. In this case, the LDA/HF ratio reduces to about 1 for $\alpha_{T}^{C}$, and to 2 for $\gamma_{T}^{C}$. Asymptotic limits (by fitting) for $\gamma_{T}^{C}$ are 8330 a.u. (LDA) and 4856 a.u. (HF). At the LDA level, the threshold of $1 \%$ deviation from the asymptotic limit is only reached at $S=1800$, that is, using $901 \mathbf{k}$ points in the BZ. The same target is achieved at the HF level using $751 \mathrm{k}$ points $(S=1500)$.

\subsection{Truncation of the exchange series}

In extended systems with small gap, the range of the density matrix (represented in the direct space) weighing the integrals of the infinite bi-electronic exchange series is widespread. Hence, the slow convergence of the optical properties with the number of terms that are included in the summation [30].

In CRYSTAL, the truncation of the infinite Coulomb and exchange series is established through five thresholds, $T_{i}(i=1 \ldots 5)$, such that integrals are disregarded (or approximated) when the overlap between the involved functions is below $10^{-T_{i}}$ (see Ref. [19] for details). Our previous work on polyacetylene [27] showed that $T_{1}, T_{2}$ and $T_{3}$ converge rapidly, as the measure of decay of the overlap of two gaussians, so that their value may safely be set to 10 . More delicate is the role of $T_{4}$ and $T_{5}$, which are associated to the exact exchange summations. We set their values according to the scheme $T_{4}=\frac{1}{2} T_{5}=T_{x}$. Table 2 reports $\alpha^{C}$ and $\gamma^{C}$ (normalized 
to one $\mathrm{C}$ ) of the CNTs $(17,0)$ and $(11,11)$ as functions of $T_{x}$. The spatial extension of the exchange interactions is indicated in terms of the maximum electronelectron distance $d_{e-e}$ for the given $T_{x}$ value. Comparison between HF and B3LYP trends is shown. As regards the $(17,0)$ tube, the two series converge pretty much in the same way despite the HF energy gap being about 3 times larger than the B3LYP one, which accounts for two orders of magnitude of difference on $\gamma_{L}^{C}$. An explanation may be that the major fraction of exact exchange in $\mathrm{HF}$ compensates the effect of the smaller gap in B3LYP. $\gamma_{L}^{C}$ requires at least $T_{x}=1500$, which corresponds to about $14 \times 10^{6}$ elements of the reducible Hamiltonian matrix to be evaluated. $\gamma_{T}^{C}$ of the conductor tube $(11,11)$ is far less sensitive to $T_{x}$ : convergence to $1 \%$ is reached at $T_{x}=200$ with B3LYP, and at $T_{x}=350$ with $\mathrm{HF}$.

\section{Results and discussion}

\subsection{Structure and energy gap}

Optimized LDA, B3LYP and HF structures of the CNTs are presented in Table 3. B3LYP provides the largest cell parameters $a$ and radii $R$; the difference with respect to the most compact geometries provided by $\mathrm{HF}$ is however very small (around $0.7 \%$ both on $a$ and $R$ ).

Let us consider now the variation of the electronic (hyper)polarizabilities with increasing the radius $R$ of the CNTs. In doing so, we will focus primarily on a comparative analysis between LDA and HF, which delimit the variation of the calculated energy gap $E_{g}$ as shown in Figure 1.

We note that for CNTs with $R<3.4 \AA$ the LDA (and B3LYP) band gap collapses with discontinuity (left panel). For larger radii, however, $E_{g}$ is found to be proportional to $1 / R$ (right panel) via a coefficient that depends on the nanotube chirality. In other words, the band gap opening occurring in graphene when the $2 D$ sheet is bent to generate a CNT depends on the rolling-up direction. The rolling-up perturbation is related to the interaction coefficient $\mathcal{G}$ that appears in the approximate expression of $E_{g}(R)$ provided by a simple $\pi$-electron model $[31,17]$,

$E_{g} \approx \frac{2 \mathcal{G}}{3 R}$

From our fittings we get: $\mathcal{G}_{3 l+1,0}=3.82$ (LDA), 5.78 (B3LYP) and $16.3(\mathrm{HF}) \mathrm{eV} \AA ; \mathcal{G}_{3 l+2,0}=6.12(\mathrm{LDA})$, 9.68 (B3LYP) and 21.5 (HF) (B3LYP) eV $\AA$. These results show that the order of effectiveness of the rollingup perturbation is $3 l+2,0>3 l+1,0$ for the different families of semiconductor CNTs, and that it is $\mathrm{HF}>>$ B3LYP > LDA for the different levels of theory.

\subsection{Electronic polarizability}

Table 4 lists the longitudinal and transverse polarizability components for the CNTs here investigated.

\subsubsection{Longitudinal component}

Upon increasing the radius by a factor 2.4, i.e., from $2.8 \AA$ for the $\operatorname{CNT}(7,0)$ to about $6.7 \AA$ for the $\mathrm{CNT}$ $(17,0)$, the longitudinal polarizability per $\mathrm{C}$ atom $\alpha_{L}^{C}$ undergoes an overall increment of about 25-29\%, with a ratio LDA/HF roughly constant around 2 . The coupledperturbed self-consistent process modify the SOS value (in parentheses) in opposite directions and by different amounts: decreases by $2 \%$ for LDA, increases by $100 \%$ for HF.

In the left panel of Figure 2, the longitudinal polarizability per unit length, $\alpha_{L}^{a}$, is plotted against $R^{2}$. Regular linear trends are shown for both LDA and HF CNTs and the fittings for the different series of semiconductors draw almost parallel lines. Moreover, for CNTs with $R \geq 3.4 \AA$ we are allowed to replace $R^{2}$ by the $R / E_{g}$ law expected for $\alpha_{L}^{a}$ (Figure 2, right panel), given the above mentioned relationship of inverse proportionality between $R$ and $E_{g}$.

One may understand such a $R / E_{g}$ law by referring to the SOS expression for the static diagonal polarizability [32], i.e.,

$\alpha_{x x}=\sum_{m \neq 0}^{\infty} \frac{x_{0 m} x_{m 0}}{\omega_{0 m}}$

where $x_{i j}=\left\langle i\left|\mu_{x}\right| j\right\rangle$ is the transition moment between the states $i$ and $j$, and $\omega_{0 j}$ is the energy of the transition between the ground state 0 and the state $j$. In case of small band gap systems such as CNTs, the fundamental transition between the top of the valence band and the bottom of the virtual band becomes predominant because $\omega_{01} \equiv E_{g} \rightarrow 0$. Therefore, Eq. 2 can be simplyfied as

$\alpha_{x x} \approx \frac{x_{01}^{2}}{E_{g}}$

The square of the transition moment at the numerator of Eq. 3, $x_{01}^{2}=x_{01} x_{10}$, expresses a probability of the transition that is inherently proportional to the number of atoms involved. Now, regarding CNTs, since the number of $\mathrm{C}$ atoms on the circumference depends directly on the radius of the tube, from Eq. 3 we retrieve the relationship $\alpha_{L}^{a} \propto R / E_{g}$.

\subsubsection{Transverse component}

The difference between the LDA and HF band structures has a negligible effect on the transverse tensor 
components. This is in agreement with earlier calculations performed in the linear regime on both semiconducting and metallic tubes $[1,33,5]$. Indeed, for all nanotubes, CP LDA and HF transverse polarizabilities $\alpha_{T}$ converge to a ratio of about 1 , Figure 3 . As regards the SOS values of $\alpha_{T}$, instead, an initial LDA/HF ratio of about 2.5 is found, which increases almost linearly with increasing $R$.

By considering the evolution of the transverse polarizability normalized for the cell parameter, $\alpha_{T}^{a}$, we find linear LDA and HF trends vs. $R^{2}$. This is in agreement with previous results obtained at the B3LYP level [7]. In that case, a fitting slope $s_{\mathrm{B} 3 \mathrm{LY}}=0.368$ was obtained, which compared well with the ideal value of $3 / 8$ derived from the classical model proposed by Bendict et al. [1], namely

$\alpha_{T}^{C P}=\frac{\alpha_{T}^{S O S}}{1+2 \alpha_{T}^{S O S} / R^{2}}$

where $\alpha_{T}^{S O S} / \alpha_{T}^{C P} \approx 4$. At the LDA level of theory, the ratio $\alpha_{T}^{S O S} / \alpha_{T}^{C P}$ increases to about 5 , yielding an ideal coefficient of $2 / 5=0.4$ that is still quite close to the fitting result, $s_{\mathrm{LDA}}=0.47$. In contrast, $\mathrm{HF}$ diverges from expectations: the slope provided by the fit is $s_{\mathrm{HF}}=$ 0.41 , to be compared with the value $1 / 4$ obtained from Eq. 4 and the observed ratio $\alpha_{T}^{S O S} / \alpha_{T}^{C P} \approx 2$.

By fitting the ratio $\alpha_{L} / \alpha_{T}$ vs. $R$ we obtain asymptotic values at about $3.5(\mathrm{SOS})$ and $18.6(\mathrm{CP})$ for LDA; at about 3.1 (SOS) and 13.5 (CP) for B3LYP; at 2.6 (SOS) and $10(\mathrm{CP})$ for HF. The CP limits turn out to be much higher than the SOS ones.

At the limit for $R \rightarrow \infty$ (i.e., the graphene slab $x y)$ it is expected that $\alpha_{L} \rightarrow \alpha_{x x}^{\text {slab }}$ and that $\alpha_{T} \rightarrow$ $(1 / 2)\left(\alpha_{x x}^{\text {slab }}+\alpha_{z z}^{\text {slab }}\right) \approx \alpha_{x x}^{\text {slab }} / 2$ (the finite value of $\alpha_{z z}^{\text {slab }}$ is negligible with respect to the infinite $\alpha_{x x}^{\text {slab }}$ value). Therefore, the ratio $\alpha_{L} / \alpha_{T}$ should be equal to 2 . However, due to the polarization the transversal field felt by the atoms in the tube is less intense than the external field (the depolarization factor along the transverse direction is $2 \pi$ ). It follows a ratio $\alpha_{L} / \alpha_{T}$ larger than 2 , particularly at the the CP level of calculation which takes better account of this effect. In HF, however, in addition to the depolarization of the transverse field $\left(\alpha_{T}^{S O S} / \alpha_{T}^{C P} \approx 2\right)$ an important effect comes also from the relaxation of the crystalline orbitals along the nanotube axis, i.e., $\alpha_{L}^{C P} / \alpha_{L}^{S O S} \approx 2$.

\subsection{Electronic second hyperpolarizability}

\subsubsection{Longitudinal component}

Table 5 reports the longitudinal second hyperpolarizabilities of semiconducting nanotubes calculated at the
LDA, B3LYP and HF levels of theory. As expected, the longitudinal component strongly depends on the energy gap (see Table 4): the LDA/HF $\gamma_{L}^{C}$ ratio ranges from 25 to 56 .

The orbitals relaxation provides a negligible contribution to the calculated LDA values of $\gamma_{L}^{C}$, the SOS/CP ratio being almost constant around 1 (see Table 5, SOS values in parentheses). In the $\mathrm{HF}$ case, on the contrary, the scale factor SOS/CP ranges from 0.15 to 0.35 . B3LYP stands midway as usual, with a ratio SOS/CP constant around 0.6 .

Figure 5 shows the trend (on a logarithmic scale) of $\gamma_{L}^{a}$ (normalized for the cell parameter) as a function of the radius for the two series of semiconducting CNTs. $(3 l+1,0)$ and $(3 l+2,0)$ CNTs show an almost linear trend for radii $R>3 \AA$ ( $\log R>0.47)$. By fitting each nanotube series with a linear function we get the slopes:

$$
\begin{aligned}
& -s_{3 l+1,0}=4.54 \text { and } s_{3 l+2,0}=6.12 \text { for LDA; } \\
& -s_{3 l+1,0}=4.41 \text { and } s_{3 l+2,0}=5.76 \text { for B3LYP; } \\
& -s_{3 l+1,0}=4.12 \text { and } s_{3 l+2,0}=5.43 \text { for HF. }
\end{aligned}
$$

The average values are 5.33 (LDA), 5.09 (B3LYP) and $4.78(\mathrm{HF})$, respectively, showing that $\gamma_{L}^{a}$ depends on the fifth power of the radius. As for $\alpha_{L}^{a}$, we can try to interpret this result looking at the SOS expression for the diagonal component of the second hyperpolarizability tensor in the static limit $[32,34]$, i.e.,

$\gamma_{x x x x}=\sum_{m, n, p \neq 0}^{\infty} \frac{x_{0 m} \bar{x}_{m n} \bar{x}_{n p} x_{p 0}}{\omega_{0 m} \omega_{0 n} \omega_{0 p}}-\sum_{m, n \neq 0}^{\infty} \frac{x_{0 m} x_{m 0} x_{0 n} x_{n 0}}{\omega_{0 m} \omega_{0 n} \omega_{0 n}}(5)$

where $\bar{x}_{i j}=\left\langle i\left|\mu_{x}\right| j\right\rangle-\delta_{i j} x_{00}$. Again, in the case of CNTs contributions other than the "fundamental" transition $0 \rightarrow 1$ may be neglected. Hence,

$\gamma_{x x x x} \approx \frac{x_{01}^{2}}{E_{g}^{3}}\left(x_{11}-x_{00}\right)^{2}-\frac{x_{01}^{4}}{E_{g}^{3}}$

By comparing Eqs. 6 and 3, we may assume

$\gamma_{x x x x} \approx \frac{\alpha_{x x}}{E_{g}^{2}}\left(x_{11}-x_{00}\right)^{2}-\frac{\alpha_{x x}^{2}}{E_{g}}$

to first order. Given that $\alpha_{L}^{a} \propto R / E_{g}$, and that $E_{g} \propto$ $1 / R$ for reasonably large CNTs, from Eq. 7 we get

$\gamma_{L}^{a} \approx A R^{4}\left(x_{11}-x_{00}\right)^{2}-B R^{5}$

where $A$ and $B$ are general proportionality coefficients. Given the positive sign of the calculated $\gamma_{L}^{a}$, the largest contibution to the second hyperpolarizability of CNTs must arise from the first term on the rhs of Eq. 8. Moreover, given that the $\left(x_{11}-x_{00}\right)^{2}$ factor is a linear combination of transition probabilities, it turns out that $\left(x_{11}-x_{00}\right)^{2} \propto R$, which finally yields $\gamma_{L}^{a} \propto R^{5}$. In order to connect the above expression with the $R^{4}$ law proposed for the third-order static dielectric susceptibility of CNTs, $\gamma_{L}$ is to be normalized for the number 
of $\mathrm{C}$ atoms in the unit cell, $n_{C}$. This corresponds to dividing Eq. 8 by $R$ (being $n_{C} \propto R$ ), which provides $\gamma_{L}^{C} \propto R^{4}$, in agreement with Eq. (28) of Ref. [17].

We may compare our analytical result with the numerical PBE estimate of $\gamma_{L}$ obtained by Kozinsky and Marzari [5] for the $(8,0)$ tube. They calculated $\gamma_{L}=$ $3.1 \times 10^{7}$ a.u. over a unit cell volume, which corresponds to $\gamma_{L}^{C}=9.7 \times 10^{5}$ a.u. per $\mathrm{C}$ atom. On our side, we optimized the structure of the CNT $(8,0)$ at the PBE level, and then performed a coupled-perturbed PBE calculation of its longitudinal hyperpolarizability, obtaining $\gamma_{L}^{C}=32 \times 10^{6}$ a.u., which reveals a second-order optical nonlinearity much larger than what Kozinsky and Marzari [5] estimated. In addition, it should be noticed that the PBE and LDA $\left(\gamma_{L}^{C}=29 \times 10^{6}\right.$ a.u. $)$ values are very close (about $8 \%$ difference), as expected for small band gap monodimensional systems [27].

Now we come to the comparison with experiments. Liu et al. [35] measured the third order dielectric susceptibility $\chi^{(3)}$ of various CNTs solutions. For a solution with $0.08 \mathrm{mg} / \mathrm{ml}$ carbon they determined the offresonant longitudinal response of the nanotubes to be $6.460 \times 10^{-14} \mathrm{esu}$; whence, an average contribution per $\mathrm{C}$ atom of about $5.921 \times 10^{-36} \mathrm{esu}$. Assuming the radius of the nanotubes in the sample to be around 5-6 $\AA$ as typical, we may draw a parallel with our calculations on the semiconducting CNTs $(13,0),(14,0),(16,0)$ and $(17,0)$, Tables 3 and 5 . Average values of $\chi_{L}^{(3)}$ per $\mathrm{C}$ atom can be obtained from those of $\gamma_{L}^{C}$ via the relation

$\chi_{L}^{(3)}=\left(\frac{2 \pi}{V} \gamma_{L}\right) c_{f}$

where $1 / V=5.95 \times 10^{-6} \mathrm{Bohr}^{-3}$ is the number of $\mathrm{C}$ atoms per unit volume as derived from the carbon mass density of the experimental sample, and $c_{f}=$ $5.038 \times 10^{-40} \mathrm{esu} / \mathrm{a} . \mathrm{u}$. is the units conversion factor [36]. The results are $\chi_{L}^{(3)}=9.340 \times 10^{-36}, 2.620 \times 10^{-36}$ and $2.261 \times 10^{-37} \mathrm{esu}$ (per C atom) at the LDA, B3LYP and HF levels of theory, respectively. We conclude therefore that, while HF underestimates the nonlinear optical response of CNTs, both density functionals provide $\chi^{(3)}$ values in good qualitative agreement with the experiment.

\subsubsection{Transverse component}

Transverse second hyperpolarizabilities are reported in Table 6 ; the order of magnitude of the calculated values is the same, both with respect to the CNT family and the Hamiltonian. At variance with respect to $\gamma_{L}, \mathrm{CP}$ results for $\gamma_{T}$ differ considerably from the SOS values (shown in parentheses).
As regards LDA, coupled-perturbed relaxation effects correct the initial SOS value by reducing it several hundreds times: the ratio SOS/CP can be as large as 850 for semiconducting CNTs and 670 for metallic CNTs. The same applies to B3LYP, although the ratio SOS/CP on $\gamma_{T}$ reduces to about 300 for both conductors and semiconductors. The CP HF correction follows the same trend (reduction of the SOS value), but in this case the ratio is around 30-50.

The transverse component $\gamma_{T}$, at variance with respect to $\gamma_{L}$, show rather small quantitative differences between LDA and $\mathrm{HF}$ and the LDA/HF ratio always remain smaller that 2. Figure 6 shows the logarithmic trends of $\gamma_{T}^{a}$ (normalized for the cell parameter) obtained for semiconducting and conducting CNTs as a function of $R$. All series of CNTs follow a linear trend. In all cases the straight lines are essentially parallel to each other (in particular looking at large $R$ values), the fitting slope being again very close to 5 .

Figure 7 shows CP ratios $\gamma_{L} / \gamma_{T}$ as functions of $R$. One can clearly see that for all Hamiltonians this ratio tends to a horizontal asymptote, unique for the two series of semiconductor CNTs, $(3 l+1,0)$ and $(3 l+2,0)$. This confirms that longitudinal and transverse second hyperpolarizabilities vary in the same way with $R^{5}$. Although being still far from the infinite radius limit, fittings provide an idea of where the asymptotes are placed at the different levels of theory. That is, between $3 \times 10^{5}$ and $6 \times 10^{5}$ for LDA, between $5 \times 10^{4}$ and $9 \times 10^{4}$ for B3LYP, between $2 \times 10^{3}$ and $4 \times 10^{3}$ for HF. We see that the choice of the Hamiltonian covers a range of uncertainty of more than two orders of magnitude. Finally, it is worth noting the large difference with respect to the asymptotes obtained for SOS results: roughly 700 (LDA), 300 (B3LYP) and 100 (HF), on average. This demonstrates the exacerbation in $\gamma$ (in comparison with $\alpha$ ) of the combined effects of field depolarization and crystalline orbitals relaxation that are involved in the coupled-perturbed process.

\section{Conclusions}

Coupled-perturbed static electronic hyperpolarizabilities of CNTs have been calculated both at the DFT (LDA, PBE and hybrid B3LYP) and HF levels of theory. This provides a large spectrum of performance in the evaluation of the energy gap and, more generally, in the description of the electronic structure. It is shown that DFT second hyperpolarizabilities yield the best agreement with the experiments, which rules out the problem of the DFT overshoot for the present case. In addition, the different density functionals are found to be equivalent in terms of qualitative accuracy. 
Predictably, the divergence of DFT methods against $\mathrm{HF}$ is found to be far more pronounced on $\gamma$ than on $\alpha$. However, the extent of such divergence depends on whether it relates with SOS estimates (unscreened or uncoupled values) obtained ahead of the self-consistent $\mathrm{CP}$ process, or with the final $\mathrm{CP}$ values corrected via the relaxation of the crystalline orbitals under the effect of the field. In fact, SOS results are generally much more far apart than converged $\mathrm{CP}$ values: the ratio $\mathrm{LDA} / \mathrm{HF}$ can be as large as 400 for $\gamma_{L}^{S O S}$ vs. 60 for $\gamma_{L}^{C P}$, and 40 for $\gamma_{T}^{S O S}$ vs. 1 for $\gamma_{T}^{C P}$. In particular, concerning $\gamma_{L}^{C P}$, we found $\mathrm{LDA} / \mathrm{HF}$ and $\mathrm{B} 3 \mathrm{LYP} / \mathrm{HF}$ ratios as large as 56 and 15 , respectively.

The amount of the CP correction varies depending on the Hamiltonian and on the direction of the field with respect to the nanotube axis. It is just about $2 \%$ (4\%) for the longitudinal second hyperpolarizability calculated at the LDA (B3LYP) level but it exceeds $600 \%$ at the HF level. DFT transverse second hyperpolarizabilities undergo a much larger variation than HF during the coupled-perturbed process: LDA and B3LYP SOS values are reduced by some hundreds times whereas the HF value is scaled only by a few tens of times.

We considered also the influence of the computational parameters on the coupled-perturbed results. Two factors play a critical role for convergence, the more so the larger the radius of the CNT. These are the number of $\mathbf{k}$ points in reciprocal space and the range of exact exchange terms considered (for HF and B3LYP). The first is relevant in particular for conducting CNTs which require up to $900 \mathrm{k}$ points to converge to better than $1 \%$ on $\gamma_{T}$. The second affects particularly $\gamma_{L}$ of semiconducting CNTs, so that the exchange series must include contributions from integrals involving electrons more than a hundred angstroms away from each other.

Finally, we determined the nonlinear optical behavior of CNTs with increasing radius. We uphold the law in $R^{4}$ predicted by Margulis et al. [17] for the macroscopic dielectric susceptibility $\chi_{L}^{(3)}$, as corresponding to the microscopic longitudinal second hyperpolarizability $\gamma_{L}^{C}$ normalized to one $\mathrm{C}$ atom. This is equivalent to an increase in $R^{5}$ of the same component normalized for the cell parameter, i.e., $\gamma_{L}^{a}$. The same trend in $R^{5}$ is found for $\gamma_{T}^{a}$, with respect to which conductor and semiconductor CNTs are essentially aligned.

These results complement and extend the present knowledge on the linear and nonlinear optical behavior of CNTs. In a future work the investigation will be extended to dynamic optical response properties of CNTs. As regards methodology, we provided a detailed analysis of the impact that the computational setting (Hamiltonian, parameters, theoretical approach) may have on the calculated results. The next step will be assessing the effect of the long-range correction to the DFT.

Acknowledgements This work, partially undertaken within the framework of CALSIMLAB, is supported by the public grant ANR-11-LABX-0037-01 overseen by the French National Research Agency (ANR) as part of the "Investissements d'Avenir" program (reference: ANR-11-IDEX-0004-02). It was granted access to the HPC resources of The Institute for scientific Computing and Simulation financed by Region Ile de France and the project Equip@Meso (reference ANR10-EQPX-29-01).

\section{References}

1. Benedict, L. X. and Louie, S. G. and Cohen, M. L., Phys. Rev. B 52, 8541 (1995)

2. Li, Y. and Rotkin, S. V. and Ravaioli, U., Nano Letters 3(2), 183 (2003)

3. Guo, G. Y. and Chu, K. C. and Wang, D.-S. and Duan, C.-G., Phys. Rev. B 69, 205416 (2004)

4. Guo, G. Y. and Chu, K. C. and Wang, D.-S. and Duan, C.-G., Comp. Mater. Sci. 30, 269 (2004)

5. Kozinsky, B. and Marzari, N., Phys. Rev. Lett. 96, 166801 (2006)

6. Brothers, E. N. and Izmaylov, A. F. and Scuseria, G. E. and Kudin, K. N., J. Phys. Chem. C $112(5), 1396$ (2008)

7. Demichelis, R. and Noël, Y. and D'Arco, Ph. and Rèrat, M. and Zicovich-Wilson, C. M. and Dovesi, R., J. Phys. Chem. C 115(18), 8876 (2011)

8. Wang, J. and Chen, Y. and Blau, W. J., J. Mater. Chem. 19, 7425 (2009)

9. Stanciu, C. and Ehlich, R. and Petrov, V. and Steinkellner, O. and Herrmann, J. and Hertel, I. V. and Slepyan, G. Y. and Khrutchinski, A. A. and Maksimenko, S. A. and Rotermund, F. and Campbell, E. E. B. and Rohmund, F., Appl. Phys. Lett. 81(21), 4064 (2002)

10. Nemilentsau, A. M. and Slepyan, G.Ya. and Khrutchinskii, A. A. and Maksimenko, S. A., Carbon 44(11), 2246 (2006)

11. Zhu, Y. and Elim, H. I. and Foo, Y.-L. and Yu, T. and Liu, Y. and Ji, W. and Lee, J.-Y. and Shen, Z. and Wee, A. T.-S. and Thong, J. T.-L., Adv. Mater. 18(5), 587 (2006)

12. Cho, W. B. and Yim, J. H. and Choi, S. Y. and Lee, S. and Schmidt, A. and Steinmeyer, G. and Griebner, U. and Petrov, V. and Yeom, D.-I. and Kim, K. and Rotermund, F., Adv. Funct. Mater. 20(12), 1937 (2010)

13. Villanueva, G. E. and Jakubinek, M. B. and Simard, B. and Oton, C. J. and Matres, J. and Shao, L.-Y. and Pérez-Millán, P. and Albert, J., Opt. Lett. 36(11), 2104 (2011)

14. Damnjanovic, M. and Milosevic, I. and Vukovic, T. and Sredanovic, R., J. Phys. A - Math. Gen. 32(22), 4097 (1999)

15. Kleinman, D. A., Phys. Rev. 126, 1977 (1962)

16. Vosko, S. H. and Wilk, L. and Nusair, M., Can. J. Phys. 58, 1200 (1980)

17. Margulis, V. A. and Sizikova, T. A., Physica B 245(2), 173 (1998)

18. Perdew, J. P. and Burke, K. and Ernzerhof, M., Phys. Rev. Lett. 77, 3865 (1996) 
19. Dovesi, R. and Saunders, V. R. and Roetti, C. and Orlando, R. and Zicovich-Wilson, C. M. and Pascale, F. and Doll, K. and Harrison, N. M. and Civalleri, B. and Bush, I. J. and D'Arco, Ph. and Llunell, M. and Causà, M. and Noël, Y., CRYSTAL14 User's Manual. Università di Torino, Torino (2014)

20. Ferrero, M. and Rérat, M. and Orlando, R. and Dovesi, R., J. Comput. Chem. 29, 1450 (2008)

21. Ferrero, M. and Rérat, M. and Orlando, R. and Dovesi, R., J. Chem. Phys. 128, 014110 (2008)

22. Ferrero, M. and Rérat, M. and Kirtman, B. and Dovesi, R., J. Chem. Phys. 129, 244110 (2008)

23. Ferrero, M. and Rérat, M. and Orlando, R. and Dovesi, R. and Bush, I., J. Phys. Conf. Ser. 117, 12016 (2008)

24. R. Orlando, V. Lacivita, R. Bast, K. Ruud, J. Chem. Phys. 132, 244106 (2010)

25. Champagne, B. and Perpéte, E. A. and van Gisbergen, S. J. A. and Baerends, E. J. and Snijders, J. G. and SoubraGhaoui, C. and Robins, K. A. and Kirtman, B., J. Chem. Phys. 109, 10489 (1998)

26. Champagne, B. and Perpéte, E. A. and van Gisbergen, S. J. A. and Baerends, E. J. and Snijders, J. G. and SoubraGhaoui, C. and Robins, K. A. and Kirtman, B., J. Chem. Phys. 110, 11664 (1999)

27. Lacivita, V. and Rérat, M. and Orlando, R. and Ferrero, M. and Dovesi, R., J. Chem. Phys. 136, 114101 (2012)

28. J. P. Perdew and A. Zunger, Phys. Rev. B 23, 5048 (1981)

29. A. D. Becke, J. Chem. Phys. 98, 5648 (1993)

30. Causà, M. and Dovesi, R. and Orlando, R. and Pisani, C. and Saunders, V. R., J. Phys. Chem. 92, 909 (1988)

31. Yorikawa, H. and Muramatsu, S., Phys. Rev. B 52, 2723 (1995)

32. Orr, B. J. and Ward, J. F., Mol. Phys. 20, 513 (1971)

33. Brothers, E. N. and Kudin, K. N. and Scuseria, G. E. and Bauschlicher, C. W., Phys. Rev. B 72, 033402 (2005)

34. Kuzyk, M. G., Phys. Rev. A 72, 053819 (2005)

35. X. Liu, J. Si, B. Chang, G. Xu, Q. Yang, Z. Pan, S. Xie, P. Ye, J. Fan, M. Wan, Appl. Phys. Lett. 74(2), 164 (1999)

36. Cox, J. D. and García de Abajo, J. F., Nature Communications 5, 5725 (2014) 


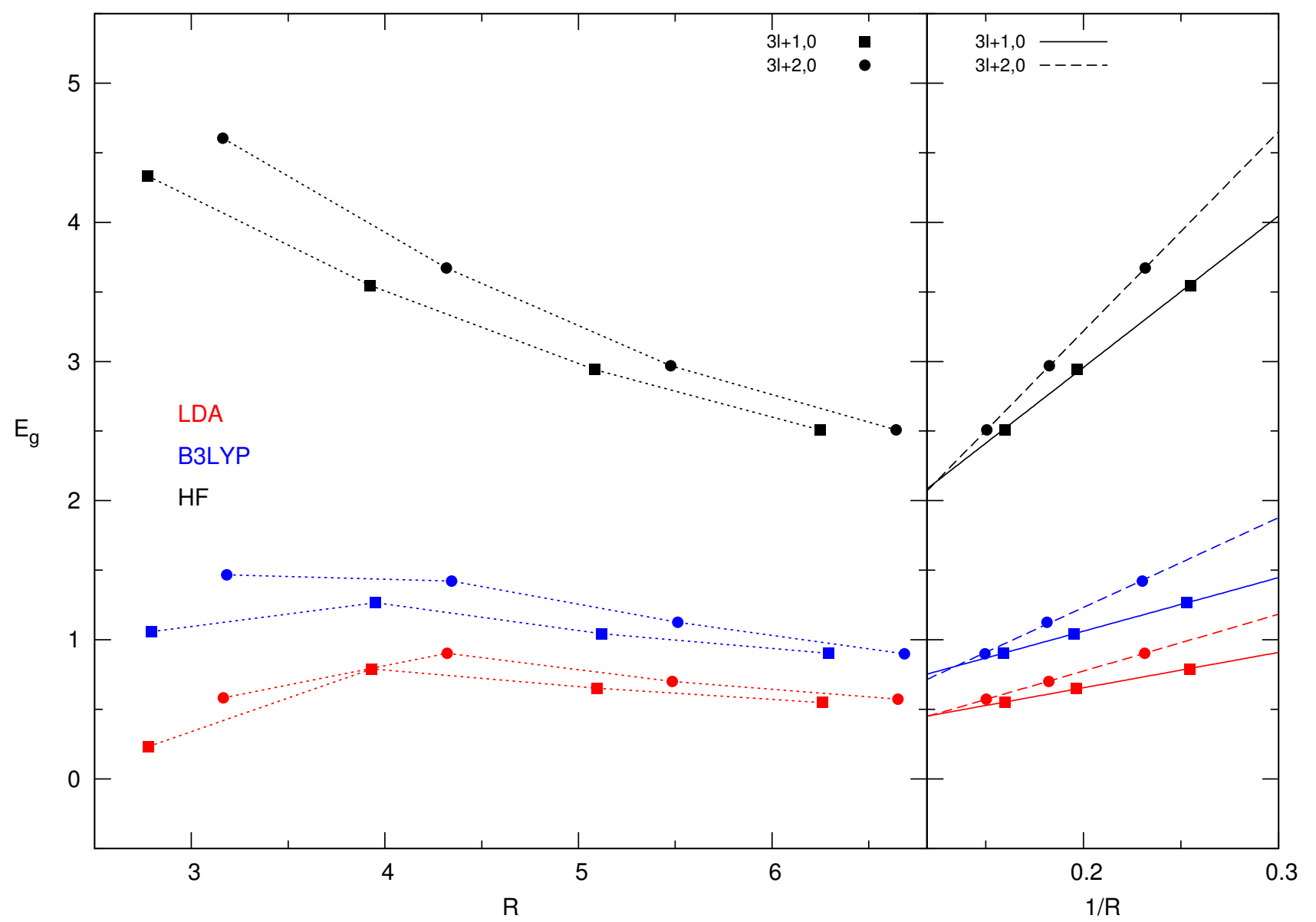

Fig. 1 Energy gap $E_{g}(\mathrm{eV})$ of semiconductor CNTs as a function of the radius $R$ ( $\AA$, on the left), and of the inverse of the radius $1 / R\left(\AA^{-1}\right.$, on the right $)$. 


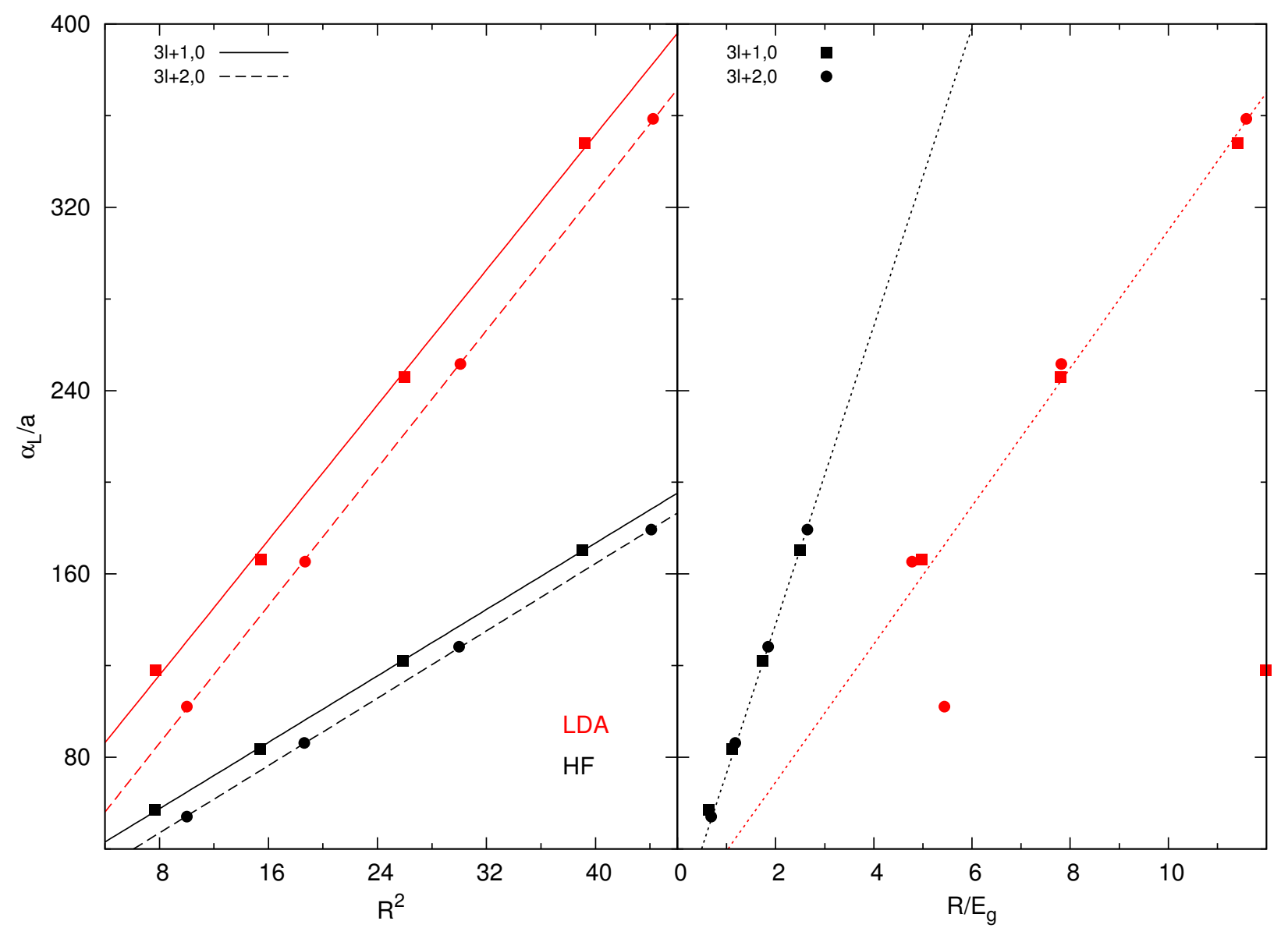

Fig. 2 Longitudinal polarizability per unit length, $\alpha_{L}^{a}\left(\AA^{2}\right)$, as a function of $R^{2}\left(\AA^{2}\right.$, left panel), and of $R / E_{g}(\AA / \mathrm{eV}$, right panel). The two LDA points lying outside the straight line (right panel) correspond to the smallest CNTs $(7,0)$ and $(8,0)$ whose band gap is in discontinuity with the trend drawn by the larger CNTs (see text). 


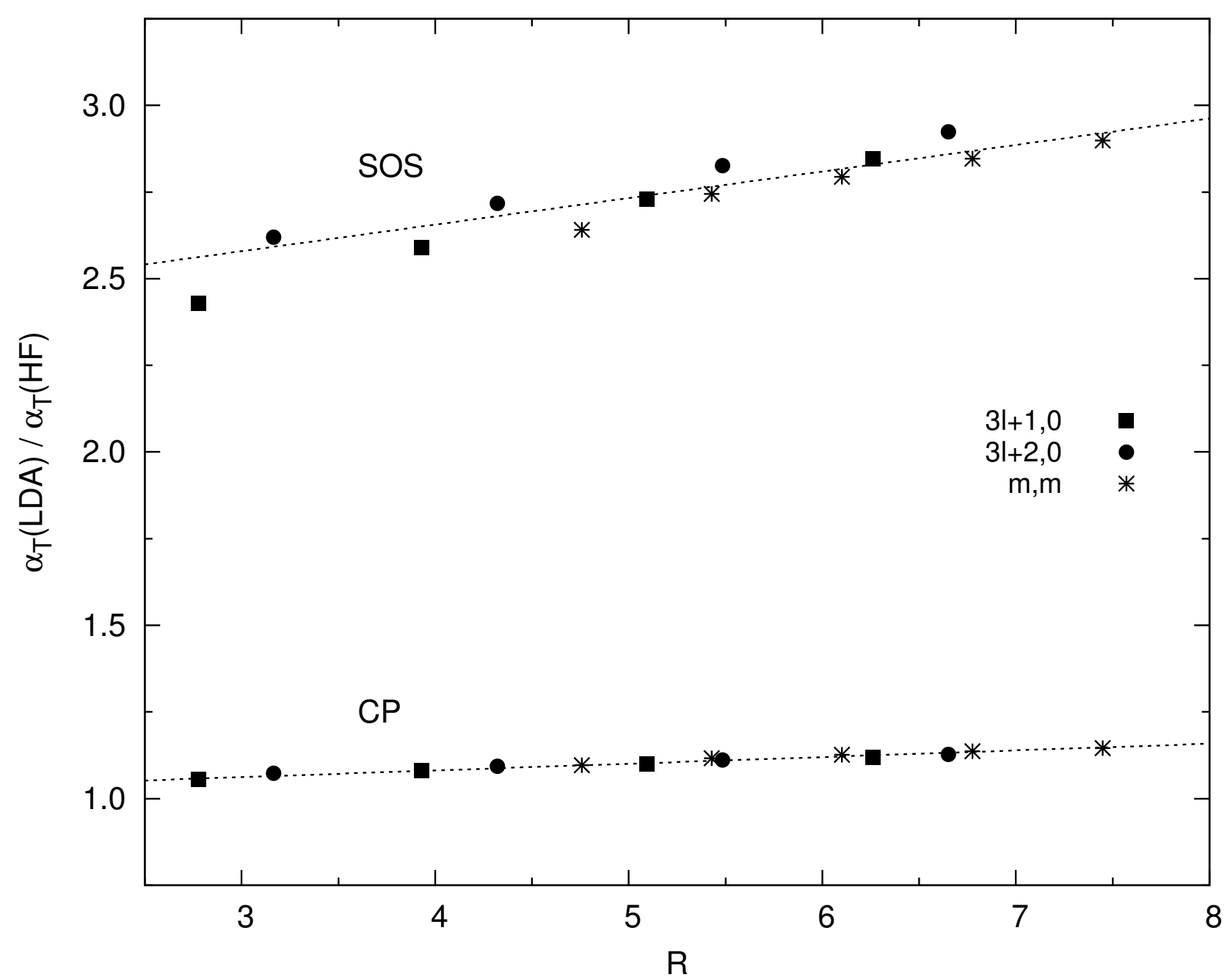

Fig. 3 LDA/HF ratio for $\alpha_{T}$ as a function of the radius $R(\AA)$. Comparison between SOS and CP trends. 


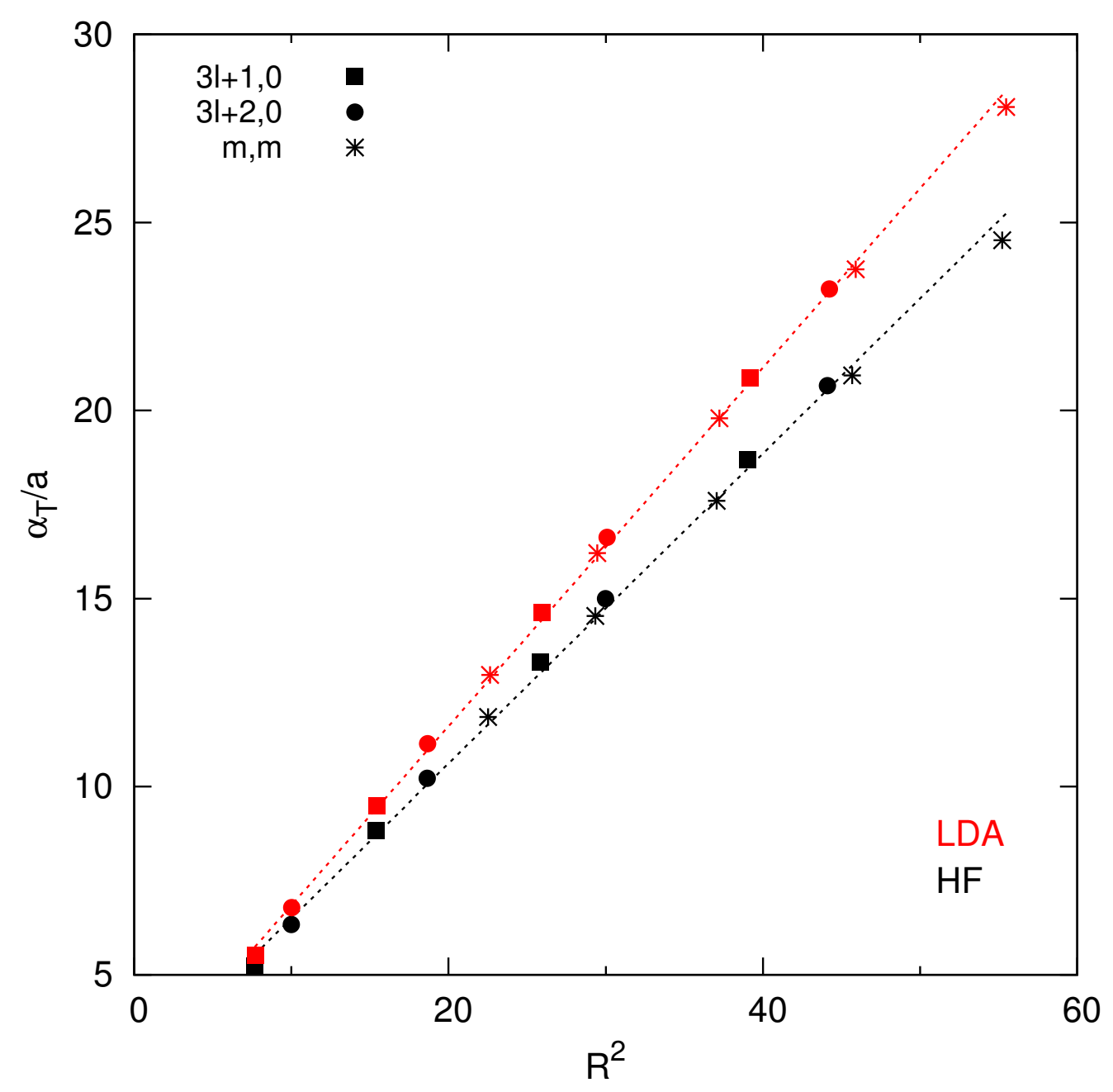

Fig. 4 Transverse polarizability per unit length, $\alpha_{T}^{a}\left(\AA^{2}\right)$, as a function of the square of the tube radius $R^{2}\left(\AA^{2}\right)$. 


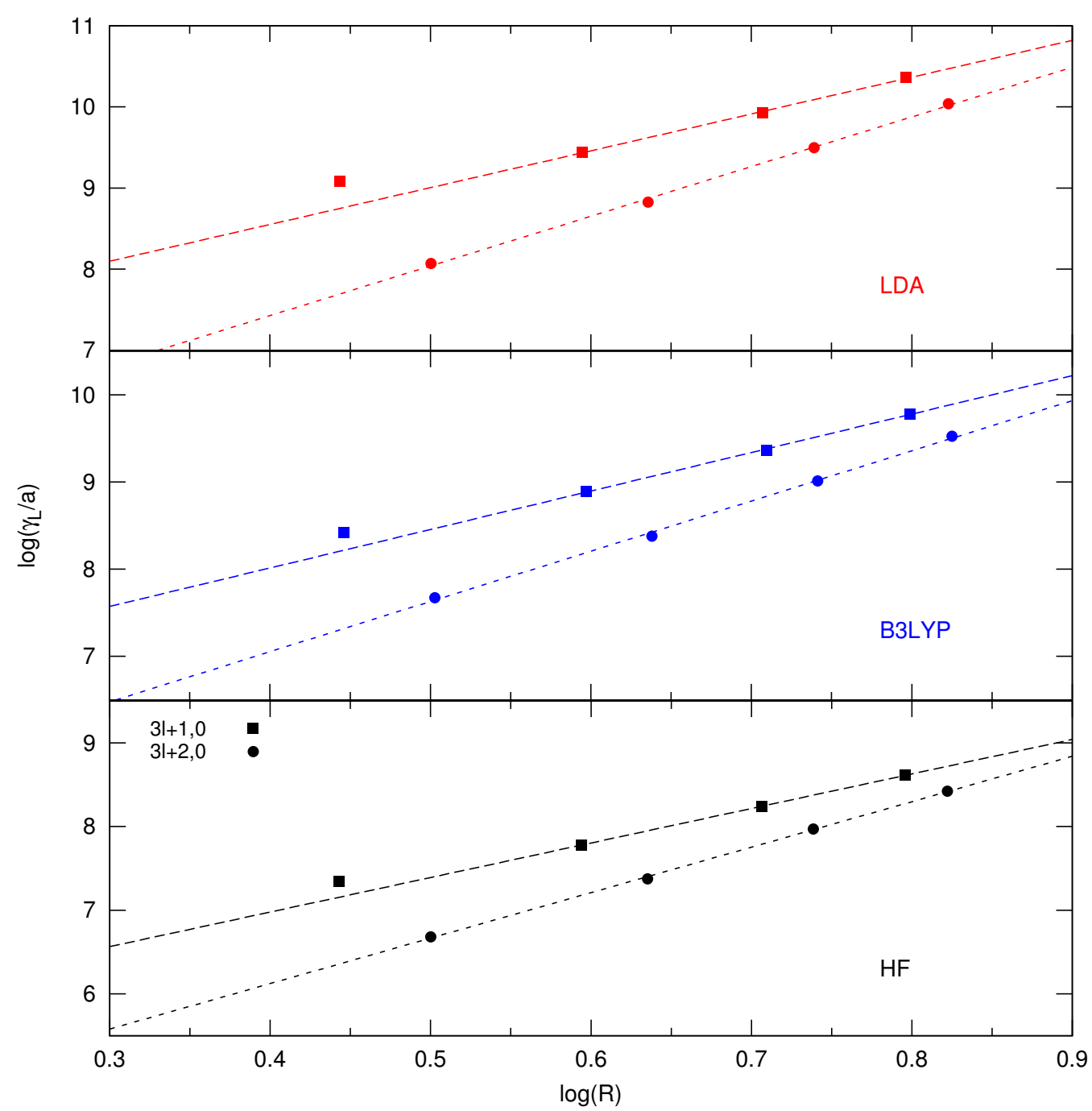

Fig. 5 Logarithmic plot of the longitudinal second hyperpolarizability per unit length, $\gamma_{L}^{a}$ (a.u.), as a function of the tube radius $R$. 


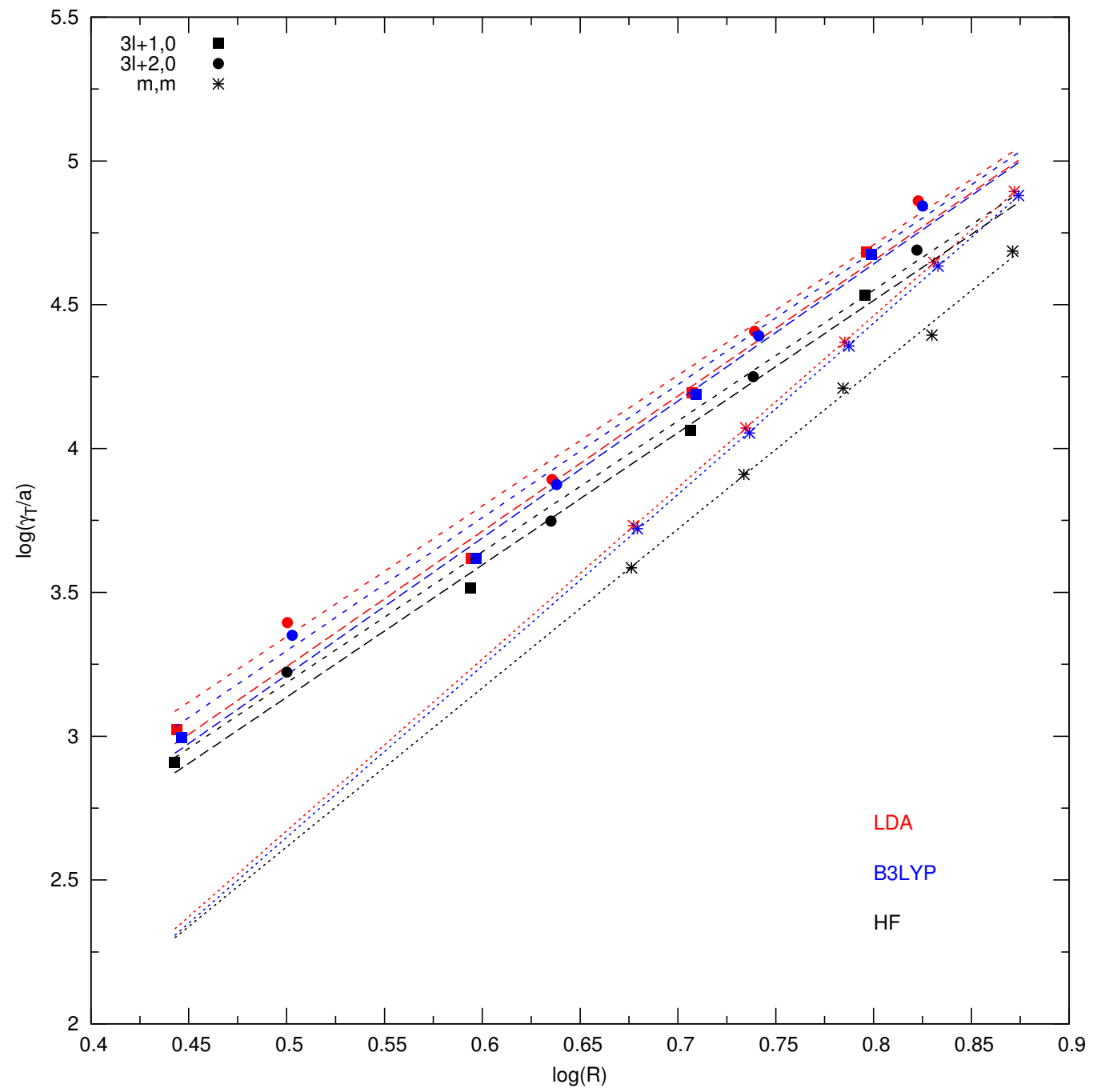

Fig. 6 Logarithmic plot of the transverse second hyperpolarizability per unit length, $\gamma_{T}^{a}$ (a.u.), as a function of the tube radius $R$. 


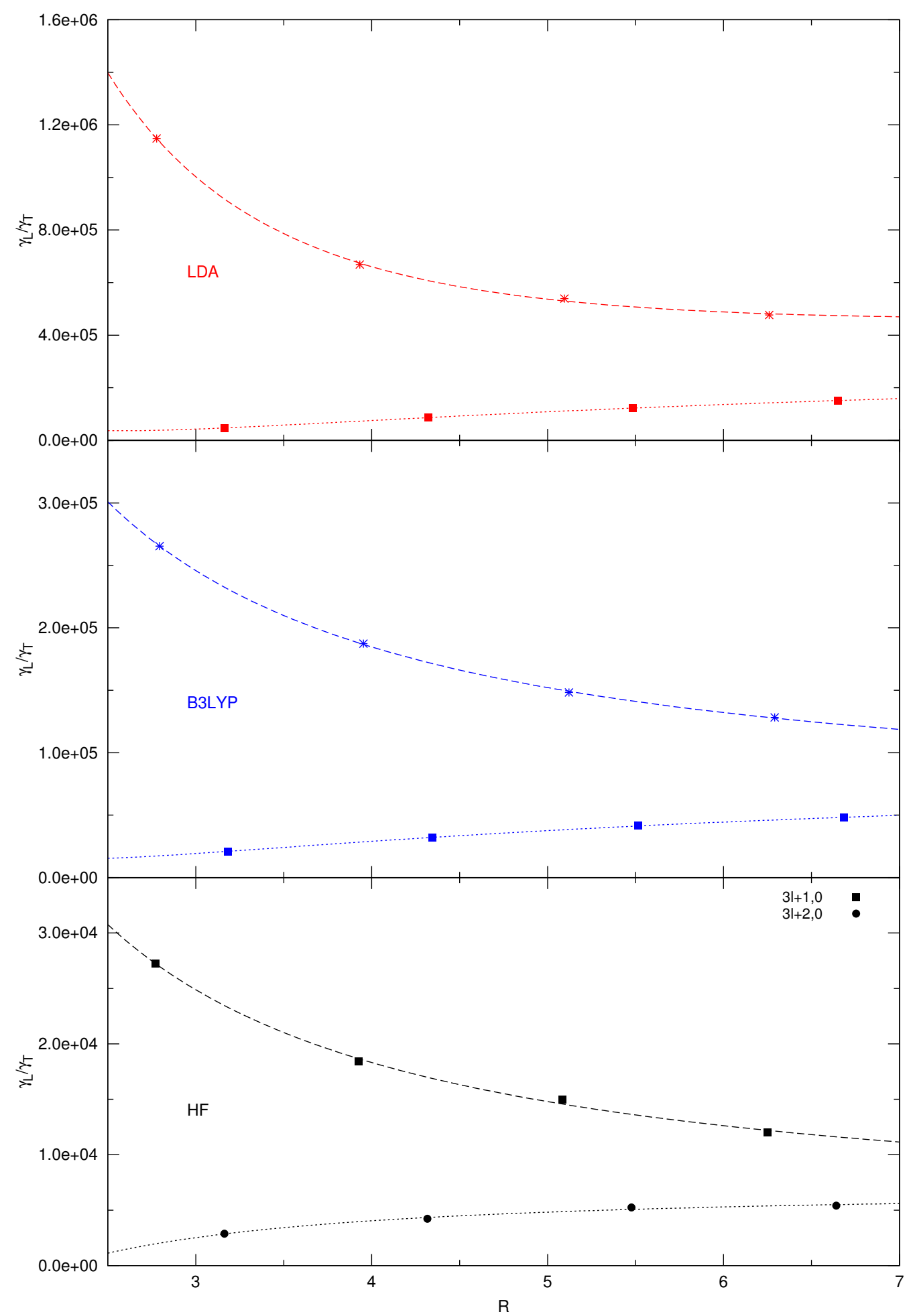

Fig. 7 Ratio between coupled-perturbed longitudinal and transverse second hyperpolarizabilities as a function of the tube radius $R$ $(\AA)$. 
Table 1 (Hyper)polarizabilities, $\alpha^{C}$ and $\gamma^{C}$ (a.u., values normalized to one C), and energy gap, $E_{g}(\mathrm{eV})$, of CNTs $(17,0)$ and $(11,11)$ as functions of the shrinking factor $S$. LDA and HF trends are compared. Note that HF $\gamma_{T}^{C}$ values for the $(11,11)$ tube are affected by some noise. Asymptotic values (by fitting) of $\gamma_{L}^{C}$ are $1.293 \times 10^{9}$ a.u. (LDA) and $2.195 \times 10^{7}$ a.u. (HF); asymptotic values of $\gamma_{T}^{C}$ are 8330 a.u. (LDA) and 4856 a.u. (HF).

\begin{tabular}{|c|c|c|c|c|c|c|c|}
\hline & \multirow[b]{2}{*}{$S$} & \multicolumn{3}{|c|}{ LDA } & \multicolumn{3}{|c|}{$\mathrm{HF}$} \\
\hline & & $\alpha^{C}$ & $\gamma^{C}$ & $E_{g}$ & $\alpha^{C}$ & $\gamma^{C}$ & $E_{g}$ \\
\hline \multirow{4}{*}{$\begin{array}{c}(17,0) \\
\alpha_{L}^{C}, \gamma_{L}^{C} \times 10^{7}\end{array}$} & 30 & 152.7 & 64.0 & 0.574 & 74.16 & 1.600 & 2.408 \\
\hline & 50 & 151.0 & 125.2 & 0.574 & 74.04 & 2.187 & 2.408 \\
\hline & 80 & 151.0 & 129.4 & 0.574 & 74.03 & 2.195 & 2.408 \\
\hline & 100 & 151.0 & 129.4 & 0.574 & 74.03 & 2.195 & 2.408 \\
\hline \multirow{9}{*}{$\begin{array}{l}(11,11) \\
\alpha_{T}^{C}, \gamma_{T}^{C}\end{array}$} & 80 & 10.53 & 9194 & 0.132 & 9.181 & 5674 & 0.500 \\
\hline & 100 & 10.58 & 6390 & 0.006 & 9.252 & 2915 & 0.237 \\
\hline & 200 & 10.56 & 7477 & 0.006 & 9.218 & 4206 & 0.138 \\
\hline & 400 & 10.55 & 7936 & 0.006 & 9.207 & 4726 & 0.089 \\
\hline & 600 & 10.55 & 8074 & 0.006 & 9.204 & 4878 & 0.072 \\
\hline & 800 & 10.55 & 8140 & 0.006 & 9.203 & 5020 & 0.059 \\
\hline & 1000 & 10.55 & 8179 & 0.006 & 9.202 & 5043 & 0.039 \\
\hline & 1500 & 10.55 & 8230 & 0.006 & 9.206 & 4813 & 0.013 \\
\hline & 1800 & 10.55 & 8246 & 0.006 & 9.204 & 4873 & 0.017 \\
\hline
\end{tabular}

Table 2 Longitudinal (hyper)polarizabilities, $\alpha_{L}^{C}$ and $\gamma_{L}^{C}$ (a.u., normalized to one C), and energy gap, $E_{g}(\mathrm{eV})$, of CNTs $(17,0)$ and $(11,11)$ as functions of the threshold $T_{x}$. Approximate electron-electron interaction maximum distances $d_{e-e}(\AA)$ are reported. Results calculated at the HF and B3LYP levels of theory are compared. Asymptotic values (by fitting) of $\gamma_{L}^{C}$ are $3.143 \times 10^{7}$ a.u. (HF) and $4.026 \times 10^{8}$ a.u. (B3LYP); asymptotic values of $\gamma_{T}^{C}$ are 5163 a.u. (HF) and 8064 a.u. (B3LYP).

\begin{tabular}{|c|c|c|c|c|c|c|c|c|}
\hline & \multirow[b]{2}{*}{$T_{x}$} & \multirow[b]{2}{*}{$d_{e-e}$} & \multicolumn{3}{|c|}{$\mathrm{HF}$} & \multicolumn{3}{|c|}{ B3LYP } \\
\hline & & & $\alpha^{C}$ & $\gamma^{C}$ & $E_{g}$ & $\alpha^{C}$ & $\gamma^{C}$ & $E_{g}$ \\
\hline \multirow{5}{*}{$(17,0)$} & 100 & 38.25 & 74.03 & 2.195 & 2.408 & 117.6 & 34.52 & 0.920 \\
\hline & 200 & 50.99 & 74.64 & 2.567 & 2.477 & 118.7 & 35.60 & 0.937 \\
\hline & 300 & 63.74 & 75.00 & 2.767 & 2.492 & 119.2 & 36.82 & 0.942 \\
\hline & 400 & 76.49 & 75.15 & 2.883 & 2.499 & 119.4 & 37.64 & 0.944 \\
\hline & 500 & 84.99 & 75.23 & 2.953 & 2.503 & 119.5 & 38.23 & 0.945 \\
\hline \multirow{5}{*}{$\alpha_{L}^{C}, \gamma_{L}^{C} \times 10^{7}$} & 600 & 89.24 & 75.28 & 3.004 & 2.505 & 119.6 & 38.63 & 0.946 \\
\hline & 800 & 106.2 & 75.31 & 3.059 & 2.507 & 119.7 & 39.18 & 0.946 \\
\hline & 1000 & 119.0 & 75.33 & 3.087 & 2.508 & 119.7 & 39.36 & 0.947 \\
\hline & 1200 & 127.5 & 75.34 & 3.102 & 2.509 & 119.7 & 39.70 & 0.947 \\
\hline & 1500 & 144.5 & 75.35 & 3.114 & 2.509 & 119.8 & 39.87 & 0.947 \\
\hline \multirow{4}{*}{$(11,11)$} & 100 & 36.83 & 9.206 & 4813 & 0.013 & 10.36 & 7917 & 0.008 \\
\hline & 200 & 51.56 & 9.220 & 4992 & 0.021 & 10.36 & 8024 & 0.008 \\
\hline & 300 & 63.84 & 9.224 & 5066 & 0.022 & 10.36 & 8051 & 0.008 \\
\hline & 350 & 68.75 & 9.226 & 5107 & 0.023 & - & - & - \\
\hline
\end{tabular}

Table 3 Optimized LDA, B3LYP and HF CNT structures. The coefficients $m$ and $n$ define the chiral vector: $(3 l+1,0),(3 l+2,0)$ and $(m, m)$ series are separated by horizontal lines. $C$ is the number of $\mathrm{C}$ atoms in the unit cell. Cell parameter $a$ and radius $R$ in $\AA$.

\begin{tabular}{|c|c|c|c|c|c|c|c|c|}
\hline \multirow[b]{2}{*}{$m$} & \multirow[b]{2}{*}{$n$} & \multirow[b]{2}{*}{$C$} & \multicolumn{2}{|c|}{ LDA } & \multicolumn{2}{|c|}{ B3LYP } & \multicolumn{2}{|c|}{$\mathrm{HF}$} \\
\hline & & & $a$ & $R$ & $a$ & $R$ & $a$ & $R$ \\
\hline 7 & 0 & 28 & 4.2438 & 2.778 & 4.2625 & 2.794 & 4.2349 & 2.772 \\
\hline 10 & 0 & 40 & 4.2458 & 3.932 & 4.2651 & 3.953 & 4.2372 & 3.925 \\
\hline 13 & 0 & 52 & 4.2459 & 5.095 & 4.2656 & 5.121 & 4.2374 & 5.085 \\
\hline 16 & 0 & 64 & 4.2458 & 6.260 & 4.2656 & 6.291 & 4.2375 & 6.248 \\
\hline 8 & 0 & 32 & 4.2407 & 3.165 & 4.2583 & 3.183 & 4.2265 & 3.163 \\
\hline 11 & 0 & 44 & 4.2433 & 4.322 & 4.2620 & 4.345 & 4.2313 & 4.317 \\
\hline 14 & 0 & 56 & 4.2441 & 5.485 & 4.2634 & 5.513 & 4.2338 & 5.477 \\
\hline 17 & 0 & 68 & 4.2445 & 6.651 & 4.2641 & 6.684 & 4.2349 & 6.641 \\
\hline 7 & 7 & 28 & 2.4504 & 4.757 & 2.4629 & 4.778 & 2.4466 & 4.746 \\
\hline 8 & 8 & 32 & 2.4506 & 5.429 & 2.4631 & 5.453 & 2.4469 & 5.416 \\
\hline 9 & 9 & 36 & 2.4508 & 6.101 & 2.4632 & 6.129 & 2.4471 & 6.087 \\
\hline 10 & 10 & 40 & 2.4509 & 6.775 & 2.4632 & 6.806 & 2.4472 & 6.759 \\
\hline 11 & 11 & 44 & 2.4509 & 7.448 & 2.4632 & 7.483 & 2.4472 & 7.432 \\
\hline
\end{tabular}


Table 4 Longitudinal $(L)$ and transverse $(T)$ polarizability components (in a.u.) normalized to one C. Comparison between coupledperturbed LDA and HF results. SOS values in parentheses. Energy gap $E_{g}$ in eV. Horizontal lines separate CNT families according to the chiral vector $(m, n)$ : series $(3 l+1,0),(3 l+2,0)$ and $(m, m)$.

\begin{tabular}{|c|c|c|c|c|c|c|c|}
\hline \multirow[b]{2}{*}{$m$} & \multirow[b]{2}{*}{$n$} & \multicolumn{3}{|c|}{ LDA } & \multicolumn{3}{|c|}{$\mathrm{HF}$} \\
\hline & & $\alpha_{L}^{C}$ & $\alpha_{T}^{C}$ & $E_{g}$ & $\alpha_{L}^{C}$ & $\alpha_{T}^{C}$ & $E_{g}$ \\
\hline 7 & 0 & $120.7(124.3)$ & $5.638(25.92)$ & 0.232 & $58.12(24.82)$ & $5.339(10.67)$ & 4.335 \\
\hline 10 & 0 & 119.1(121.0) & $6.810(32.68)$ & 0.791 & $59.79(28.25)$ & $6.304(12.62)$ & 3.546 \\
\hline 13 & 0 & $135.6(137.1)$ & $8.061(39.65)$ & 0.653 & $67.18(32.74)$ & $7.324(14.53)$ & 2.940 \\
\hline 16 & 0 & $155.8(157.1)$ & $9.340(46.62)$ & 0.548 & $76.03(37.42)$ & $8.353(16.39)$ & 2.508 \\
\hline 8 & 0 & $91.28(91.78)$ & $6.065(29.46)$ & 0.582 & $48.19(25.80)$ & $5.650(11.24)$ & 4.604 \\
\hline 11 & 0 & $107.6(108.3)$ & $7.251(35.75)$ & 0.904 & $55.95(29.88)$ & $6.631(13.16)$ & 3.672 \\
\hline 14 & 0 & $128.7(129.5)$ & $8.504(42.51)$ & 0.702 & $65.40(34.50)$ & $7.653(15.05)$ & 2.970 \\
\hline 17 & 0 & $151.0(151.9)$ & $9.786(49.39)$ & 0.574 & $75.34(39.23)$ & $8.682(16.90)$ & 2.509 \\
\hline 7 & 7 & - & $9.571(36.68)$ & 0.006 & - & $6.987(13.89)$ & 0.442 \\
\hline 8 & 8 & - & $6.704(40.30)$ & 0.002 & - & $7.501(14.68)$ & 0.521 \\
\hline 9 & 9 & - & $9.091(43.75)$ & 0.005 & - & $8.070(15.66)$ & 0.568 \\
\hline 10 & 10 & - & $9.818(47.30)$ & 0.006 & - & $8.639(16.62)$ & 0.604 \\
\hline 11 & 11 & - & $10.55(50.90)$ & 0.006 & - & $9.205(17.56)$ & 0.634 \\
\hline
\end{tabular}

Table 5 Longitudinal second hyperpolarizabilities of semiconductor CNTs normalized to one C, $\gamma_{L}^{C}\left(10^{6}\right.$ a.u.). Comparison between coupled-perturbed LDA, B3LYP and HF results. SOS values in parentheses. CNT series $(3 l+1,0)$ and $(3 l+2,0)$ are separated by a horizontal line.

\begin{tabular}{|c|c|c|c|}
\hline$m, 0$ & LDA & B3LYP & $\mathrm{HF}$ \\
\hline 7 & $346.42(351.57)$ & $75.357(54.986)$ & $6.3080(0.9364)$ \\
\hline 10 & $557.53(562.51)$ & $156.68(96.968)$ & $12.053(2.3808)$ \\
\hline 13 & 1297.1 & $354.29(222.58)$ & $26.593(6.1150)$ \\
\hline 16 & $2891.4(2905.6)$ & $764.98(486.15)$ & $51.342(13.997)$ \\
\hline 8 & $29.378(29.476)$ & $11.856(7.9576)$ & $1.1983(0.4496)$ \\
\hline 11 & $122.52(122.25)$ & $43.830(28.232)$ & $4.2954(1.4512)$ \\
\hline 14 & $451.21(450.52)$ & $147.76(93.958)$ & $13.322(4.3447)$ \\
\hline 17 & $1293.9(1292.8)$ & $397.30(252.63)$ & $31.212(10.893)$ \\
\hline
\end{tabular}

Table 6 Transverse second hyperpolarizabilities normalized to one C, $\gamma_{T}^{C}$ (10 $20^{2}$ a.u.). Comparison between coupled-perturbed LDA, B3LYP and HF results. SOS values in parentheses. Horizontal lines separate CNT families according to the chiral vector $(m, n):$ series $(3 l+1,0),(3 l+2,0)$ and $(m, m)$.

\begin{tabular}{|c|c|c|c|c|}
\hline$m$ & $n$ & LDA & B3LYP & $\mathrm{HF}$ \\
\hline 7 & 0 & $3.0164(2084.5)$ & $2.8393(755.13)$ & $2.3133(79.088)$ \\
\hline 10 & 0 & $8.3345(6207.8)$ & $8.3637(2493.5)$ & $6.5422(259.42)$ \\
\hline 13 & 0 & 24.071 (18555.) & $23.907(7214.3)$ & $17.759(704.77)$ \\
\hline 16 & 0 & 60.607 (47415.) & 59.672 (17714.) & 42.799 (1631.4) \\
\hline 8 & 0 & $6.2188(5306.4)$ & $5.6405(1639.9)$ & 4.1663 (139.81) \\
\hline 11 & 0 & 14.219 (11611.) & $13.731(4293.5)$ & $10.170(399.71)$ \\
\hline 14 & 0 & 36.617 (29759.) & 35.441 (10973.) & $25.376(995.88)$ \\
\hline 17 & 0 & $85.612(69390)$. & $82.676(24920)$. & $57.697(2173.5)$ \\
\hline 7 & 7 & $19.366(6086.0)$ & $8.7476(2831.5)$ & $6.3627(339.26)$ \\
\hline 8 & 8 & 17.053 (11470.) & $16.477(5079.5)$ & $11.754(580.76)$ \\
\hline 9 & 9 & 30.118 (20183.) & $29.411(8656.0)$ & $20.855(940.11)$ \\
\hline 10 & 10 & 51.342 (33956.) & $50.132(14110)$. & $28.673(1454.5)$ \\
\hline 11 & 11 & 82.464 (53970.) & $80.240(21860)$. & $51.069(2172.5)$ \\
\hline
\end{tabular}

